\title{
El valor pedagógico de Telegram como complemento del mobile learning en la formación en finanzas: aplicación práctica a un caso de estudio
}

\author{
Javier de los Ríos Medina \\ Doctor en Educación y Nuevas Tecnologías/Experto en mobile learning \\ delosriosmedina@gmail.com | https://orcid.org/0000-0002-8707-3397
}

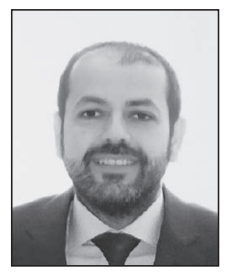

Este trabajo ha obtenido el 1. er Premio Estudios Financieros 2020 en la modalidad de Educación y Nuevas Tecnologías.

El jurado ha estado compuesto por: don Manuel Area Moreira, doña Eva María Bailén Fernández, don Julio Cabero Almenara, doña Ana García-Valcárcel Muñoz-Repiso, don Pedro José González Felipe y don Alfonso Gutiérrez Martín.

Los trabajos se presentan con seudónimo y la selección se efectúa garantizando el anonimato de los autores.

\section{Extracto}

Esta investigación analiza el valor pedagógico de Telegram como complemento para el mobile learning en la formación en un curso de finanzas personales en el que participaron 1.205 alumnos.

La base teórica se sustenta sobre los constructos desarrollados en la teoría de aceptación de la tecnología (technology acceptance model [TAM]) y en los 10 criterios de Nokelainen en relación con la usabilidad pedagógica.

La pregunta de investigación relaciona positivamente el uso de Telegram con los modelos de aprendizaje, la labor de los docentes, la interrelación de los alumnos, la importancia de los contenidos y el uso de los dispositivos.

Las conclusiones son muy positivas, ya que Telegram destaca por su alto grado de satisfacción en relación con el modelo de aprendizaje -con una tasa de abandono máxima del 2,90\%-, es un magnífico complemento para el desempeño de los docentes y se incrementa su potencial con el empleo de contenidos audiovisuales y la utilización de multidispositivos indistintamente.

Palabras clave: Telegram; mobile learning; modelos de aprendizaje; usabilidad pedagógica; usabilidad técnica; teoría de aceptación de la tecnología (technology acceptance model [TAM]); vídeo; chatbot; e-learning; innovación docente; aprendizaje basado en problemas (problems based learning [PBL]); transformación digital; WhatsApp; WeChat; transformación social.

Fecha de entrada: 01-06-2020 / Fecha de aceptación: 18-09-2020

Cómo citar: Ríos Medina, J. de los. (2021). El valor pedagógico de Telegram como complemento del mobile learning en la formación en finanzas: aplicación práctica a un caso de estudio. Tecnología, Ciencia y Educación, 18, 7-42. 


\title{
The pedagogical value of Telegram as a complement to mobile learning in finance training: practical application to a case study
}

\author{
Javier de los Ríos Medina
}

\section{Abstract}

This research investigates the pedagogical value of Telegram as a complement to mobile learning in the context of a personal finance course in which 1,205 students participated.

The theoretical basis is based on the constructs developed in the acceptance theory of technology acceptance model (TAM) and in the 10 Nokelainen criteria in relation to pedagogical usability.

The research question positively relates the use of Telegram to learning models, the work of teachers, the interrelationship of students, the importance of content and the use of devices.

The study's conclusions are very positive, as Telegram stands out for its high degree of satisfaction in relation to the learning model, with a maximum dropout rate of $2.90 \%$, it is a magnificent complement to the performance of teachers and increases their potential with the use of audiovisual content and the use of multi-devices interchangeably.

Keywords: Telegram; mobile learning; learning models; pedagogical usability; technical usability; technology acceptance model (TAM); video; chatbot; e-learning; teaching innovation; problems based learning (PBL); digital transformation; WhatsApp; WeChat; social transform. 


\section{Sumario}

1. Introducción

1.1. Propósito de la investigación

1.2. Justificación de la investigación

1.3. Objetivos

1.4. Proposiciones de investigación

2. Marco referencial

2.1. El origen de Telegram

2.2. Características de Telegram

2.3. Telegram como herramienta de mobile learning

3. Marco teórico

\subsection{Modelo TAM}

3.2. Modelos de usabilidad pedagógica

3.3. Estudio del caso: población, muestra y diseño del cuestionario

3.4. Descripción y contenido del curso de finanzas personales

4. Resultados de la investigación

4.1. Resultados cuantitativos

4.2. Resultados cualitativos

5. Análisis de resultados

5.1. Análisis cuantitativo

5.2. Análisis cualitativo

6. Principales hallazgos de la investigación

6.1. El valor pedagógico de Telegram y la metodología de aprendizaje

6.2. El valor pedagógico de Telegram y los docentes

6.3. El valor pedagógico de Telegram y la interrelación entre los alumnos

6.4. El valor pedagógico de Telegram y los contenidos

6.5. El valor pedagógico de Telegram y los dispositivos

7. Conclusiones

8. Reflexión final

Referencias bibliográficas 


\section{Introducción}

En el año 2020 no ha habido ningún sector de la economía o del país que no se haya visto obligado a reflexionar sobre sus modelos de negocio y sobre la relación con sus empleados, sus proveedores o sus clientes. En definitiva, sobre su supervivencia o las posibles oportunidades que puede depararnos esta situación de la COVID-19.

El sector de la formación ha sido uno de los que antes ha entendido la necesidad de dar soluciones y respuestas a sus alumnos en el corto plazo. Al tiempo, debe reflexionar sobre el impacto a medio y largo plazo de la dualidad entre la formación presencial y online, la importancia de la tecnología en la formación y los nuevos métodos de aprendizaje que deberán desarrollarse.

\section{El sector de la formación debe reflexionar sobre el impacto a medio y largo plazo de la dualidad entre la formación presencial y online, sobre la importancia de la tecnología en la formación y sobre los nuevos métodos de aprendizaje que deberán desarrollarse}

\subsection{Propósito de la investigación}

Este estudio ve la luz en un momento donde es necesario evaluar la importancia de las tecnologías para el aprendizaje, reflexionar sobre su uso y su valor real, más allá de una posible moda, y saber cómo aplicarlas en este nuevo paradigma.

El propósito principal de este trabajo es analizar y evaluar el valor pedagógico de Telegram como complemento para el mobile learning en la formación a través de un curso de finanzas personales.

La pregunta de la investigación es la siguiente:

¿En qué medida el uso de nuevas herramientas tecnológicas aporta valor a la formación a través de dispositivos móviles?

Es una pregunta que aborda diferentes aspectos de la formación, como la metodología de aprendizaje, la labor de los docentes, el papel de los alumnos, la importancia de los contenidos o el uso de diferentes canales o dispositivos. 


\subsection{Justificación de la investigación}

\section{A) La transformación digital como transformación social}

La transformación digital ya no solo se considera una transformación en la tecnología o en las innovaciones, sino que se entiende como una transformación social. Esa transformación social puede desarrollarse a través de un proceso de adaptación a esa nueva tecnología, acelerado por las mejoras en la usabilidad de las aplicaciones y a través de los cambios sociales que se van produciendo.

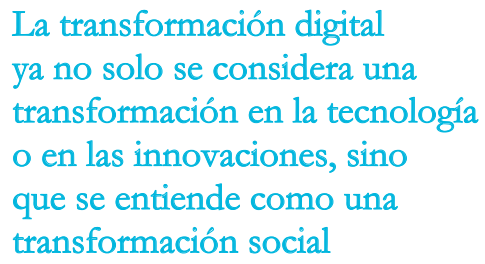

La transformación digital ya no solo se considera una transformación en la tecnología o en las innovaciones, sino que se entiende como una transformación social

La transformación digital está presente en nuestras vidas, en nuestras empresas y en la forma en que nos relacionamos y modificamos nuestros hábitos como consumidores, usuarios o clientes a través del uso y de la interacción con diferentes tipos de dispositivos hasta llegar a los dispositivos móviles.

El creciente consumo de medios móviles y el desarrollo de la tecnología de estos dispositivos están provocando que el consumo de móvil presente crecimientos exponenciales, como se puede ver en el Estudio Anual de Mobile \& Conected Devices de IAB (marzo 2019)1.

Ello ha tenido un gran efecto en todos los sectores y, también, en la forma de enfocar la enseñanza y el aprendizaje a través de dispositivos móviles o mobile learning, como se desprende del Informe Horizon $2019^{2}$.

\section{B) La creciente importancia del mobile learning}

Son múltiples las fuentes y los estudios que pronostican el crecimiento exponencial de esta tecnología. A modo de ejemplo, se destacan algunas previsiones para el 2025 -en el Global Mobile Learning Market Size and Forecast to 2025- que ayudan a contextualizar la importancia del mobile learning.

La United Nations Educational, Scientific and Cultural Organization (UNESCO), en su informe Turning on Mobile Learning Europe: Illustrative Initiatives and Policy Implications

\footnotetext{
1 https://iabspain.es/wp-content/uploads/estudio-anual-mobile-connected-devices-2019_iab-spain_ vreducida.pdf

2 https://library.educause.edu/ /media/files/library/2019/2/2019horizonreportpreview.pdf
} 
$(2012)^{3}$, que es el informe más recientemente publicado, hace referencia a la importancia de las disciplinas relacionadas con la formación online y, en concreto, con el mobile learning. Insta a las organizaciones a desarrollar programas y nuevas metodologías que contribuyan a mejorar el acceso a la formación y a la igualdad de oportunidades.

\section{C) Escasez de estudios sobre Telegram como herramienta de mobile learning}

Las aplicaciones móviles son una herramienta de gran valor para el sector de la formación, pero el número de estudios y la profundidad son insuficientes.

Esta escasez de estudios es analizada por Sivabalan y Ali (2019) a partir de la investigación realizada por Garrote et al. (2017). En este trabajo, se seleccionan 107 estudios sobre aplicaciones móviles utilizadas en formación. Los resultados muestran que solo 4 (10\%) pertenecen a Telegram, uno a WeChat (2,50 \%), uno a LINE (2,50\%) y los 34 restantes (85\%) a WhatsApp.

\section{D) Oportunidades para la formación online}

Desde la aparición de la COVID-19, muchos han sido los efectos económicos y sociales sobre el sector formación tanto en su modalidad presencial como en su versión online.

A corto plazo, las principales consecuencias sobre la formación ocasionadas por la COVID-19 se concentran en tres aspectos que se destacan a continuación:

- La imposibilidad de impartir formación presencial.

- Los cambios sociales que han impactado en la forma en la que recibimos la formación.

- El uso de la tecnología que ha facilitado nuevos canales y herramientas de formación que complementan (o, posiblemente, sustituyen) a la formación presencial y a la formación online.
A corto plazo, las principales
consecuencias sobre la formación ocasionadas por la COVID-19
se concentran en tres aspectos:
la imposibilidad de impartir
formación presencial, los cambios
sociales que han impactado en
la forma en la que recibimos la
formación y el uso de la tecnología
que ha facilitado nuevos canales
y herramientas de formación que
complementan (o, posiblemente, sustituyen) a la formación
presencial y a la formación online

3 http://unesdoc.unesco.org/images/0021/002161/216165E.pdf 
Las primeras reflexiones nos conducen a la idea de que han surgido nuevos desafíos para las universidades, las escuelas de negocio, las empresas de tecnología y los docentes. Es aún pronto para cuantificar los efectos económicos, que no serán en todos los casos positivos, y ver si realmente se ha producido un cambio de paradigma en el alumno a favor de la formación online en cualquiera de sus variantes.

\subsection{Objetivos}

El objetivo principal de este trabajo es analizar el valor pedagógico de Telegram como complemento para el mobile learning aplicado a un caso de estudio. Este análisis se va a realizar a través del estudio de uno de los constructos más representativos de las teorías de aceptación de la tecnología, como es la facilidad de uso o usabilidad. Por tanto, el objetivo es analizar la usabilidad de Telegram desde una perspectiva pedagógica.

Como en toda investigación, el objetivo principal está condicionado por objetivos secundarios, que son:

- Estudiar y actualizar las teorías relacionadas con los modelos de aceptación de la tecnología y destacar el modelo que más pueda adaptarse al caso de estudio.

- Avanzar en el análisis del mobile learning a través de la experimentación con una aplicación de comunicación multidispositivo como Telegram.

- Analizar los resultados metodológicos de un curso de finanzas personales impartido mediante el canal móvil.

\subsection{Proposiciones de investigación}

Las proposiciones de investigación se han diseñado pensando en cinco conceptos fundamentales que estudia la usabilidad pedagógica de una aplicación: los métodos de aprendizaje, la labor de los docentes, la interrelación entre los alumnos, la importancia de los contenidos y el uso de los dispositivos.

Por tanto, la usabilidad pedagógica de Telegram se analiza desde el prisma de estas cinco proposiciones ${ }^{4}$ que se enumeran a continuación:

\section{Las proposiciones de investigación se han diseñado pensando en cinco conceptos fundamentales que estudia la usabilidad pedagógica de una aplicación: los métodos de aprendizaje, la labor de los docentes, la interrelación entre los alumnos, la importancia de los contenidos y el uso de los dispositivos}

4 En los apartados relativos a los resultados y al análisis de los mismos se citan como «P», «P1», «P2», etc. 
- P1. Modelos de aprendizaje. Telegram facilita el desarrollo de modelos de aprendizaje.

- P2. Docentes. Telegram mejora los resultados de los docentes a través de los dispositivos móviles.

- P3. Alumnos. Telegram facilita la interrelación entre los alumnos a través de los dispositivos móviles.

- P4. Contenidos. Incorporar contenidos audiovisuales en Telegram mejora la usabilidad pedagógica.

- P5. Canales. Compaginar diferentes dispositivos móviles (teléfono móvil y tablet o teléfono móvil y portátil o PC) mejora la usabilidad pedagógica.

\section{Marco referencial}

El marco referencial del presente trabajo podría estructurarse alrededor de conceptos tan amplios como el de «innovación», «tecnología móvil» o «transformación digital», como se ha comentado en el apartado 1. Sin embargo, entendemos necesario centrar la investigación en aspectos más concretos y, por tanto, se ha preferido delimitar el marco referencial en torno a Telegram, sus características más representativas y su aplicación al mobile learning.

\subsection{El origen de Telegram}

Telegram es una aplicación de comunicación móvil diseñada y desarrollada por los hermanos Pavel y Nikolai Durov (fundadores de la red social rusa VKontakte), que permite a los usuarios comunicarse y enviar contenidos en formato de archivos, audio o vídeo a través de smartphones, tablets u ordenadores de sobremesa de forma síncrona.

En relación con la compañía, Telegram tiene su sede en Berlín y en abril de 2020 contaba con 400 millones de usuarios. La versión para IOS se comercializó el 14 de agosto de 2013 y, para Android, el 20 de octubre de 2013. A nivel tecnológico, está considerada como una de las aplicaciones más seguras que se comercializan a nivel usuario.

\subsection{Características de Telegram}

Como puede verse a continuación, el cuadro 1 contiene un resumen con las características más importantes de Telegram en relación al mobile learning y a las cinco proposiciones enumeradas en el apartado 1.4 . 


\section{Cuadro 1. Características de Telegram en relación al mobile learning y a las cinco proposiciones de investigación}

Características

\section{Enumeración de características}

Características de Telegram para el mobile learning relacionadas con el aprendizaje.
Formación de tipo blended, programación de tareas, evaluación de los alumnos a través de cuestionarios propios, trazabilidad del proceso de aprendizaje, ubicuidad, innovación metodológica, planteamiento de problemas a resolver, atención activa de los alumnos, seguimiento, nueva experiencia, retroalimentación inmediata, mayor calidad del proceso formativo y automatización.

Características de Telegram Administrador de la aplicación, visualización de contenidos, trazabilidad, historial único de conversaciones, posibilidad de estar varios docentes en el aula, posibilidad de generar contenidos conversacionales y facilidad para creación de grupos segmentados.

Características de Telegram para el mobile learning relacionadas con el alumno.

Seguridad, privacidad, cercanía con el alumno, posibilidad de compaginar estudios con trabajo y familia, participación activa, flexibilidad, participación asíncrona o síncrona, universalidad, posibilidad de silenciar notificaciones, costo, eficiencia en la comunicación, chats secretos, alias público y acceso al contenido de forma ilimitada.

Características de Telegram Borrado y edición de mensajes, intercambio de archivos de hasta 1,5 GB para el mobile learning relacada uno, emoticonos como forma de comunicación informal, automatización, autodestrucción de la cuenta de Telegram, mensajes en la nube, variedad de contenidos, incorporación de hashtag, packs externos de idiomas, invitación a través de un link de acceso, acceso ilimitado a los mensajes y sin conexión, búsqueda rápida, posibilidad de exportar todos los datos y variedad de formularios.

Características de Telegram Repositorio de contenidos, canales ilimitados, multidispositivo, sincronización, coste, notificaciones inteligentes, multiplataforma y multidispositivo. Hay que observar que muchas características se repiten en otras categorías.

\subsection{Telegram como herramienta de mobile learning}

A pesar de que el número de estudios sobre Telegram como herramienta para el mobile learning es escaso, se han seleccionado una relación de los nueve más interesantes y recientes, como puede verse en el cuadro 2. 


\section{Cuadro 2. Estudios sobre Telegram como herramienta para el mobile learning}

Estudio/Autor
Effectiveness of the use of in-
tegrated project based learning
model, Telegram messenger,
and plagiarism checker on lear-
ning outcomes (Pratama y Pra-
styaningrum, 2019).

Mobile learning: innovation in teaching and learning using Telegram (Iksan y Saufian, 2017).

The impact of mobile learning on ESP learners' performance (Alkhezzi y Al-Dousari, 2016).

The effectiveness of social media network Telegram in teaching English language pronunciation to Iranian EFL learners (Xodabande, 2017).

M-learning-Una experiencia colaborativa usando el software Telegram (Salas y Subía, 2018).

Usos de las aplicaciones móviles de mensajería en la docencia universitaria: Telegram (Martínez et al., 2017).

Maximizing Telegram application for teaching reading (Sari, 2017).

Microlearning for macro-outcomes: students' perceptions of Telegram as a microlearning tool (Aldosemani, 2019).

Contenido

\section{Conclusiones}

Estudia la validez de los modelos de PBL en los que se incorpora Telegram como el canal principal.
El aprendizaje mejora con Telegram como elemento conector entre aprendizaje presencial y en línea.
Esta investigación explora la aplicación Telegram en los procesos de enseñanza y aprendizaje.

El impacto de Telegram en la enseñanza y el aprendizaje de la lengua inglesa.
Esta investigación estudia cómo enseñar la pronunciación del inglés a alumnos iraníes en un curso de lengua extranjera a través de Telegram.

Cómo enseñar a través de Telegram con una metodología de aprendizaje basada en una experiencia colaborativa.

Estudia cómo los docentes pueden aprovechar Telegram para mejorar sus propuestas formativas.

Estudia el uso de Telegram en un curso en el que se fomenta la lectura en inglés como parte del aprendizaje.

Estudia la efectividad del microaprendizaje desde la perspectiva de los alumnos a través de Telegram.
Telegram aporta a los alumnos una nueva experiencia, más creativa y más inclusiva y espontánea.

Telegram aporta a los alumnos un impacto positivo en la comprensión del vocabulario y de las reglas gramaticales.

Las conclusiones son que el aprendizaje de idiomas, en concreto la pronunciación, mejora con el uso de aplicaciones móviles de comunicación.

Telegram permite que los alumnos estén atentos a las actividades del grupo y a la interacción entre ellos y con los docentes.

Hay dos vertientes diferenciadas: la funcionalidad como canal de comunicación y la gestión de bots.

El aprendizaje de idiomas y, en concreto, la lectura mejoran con el uso de aplicaciones móviles de comunicación como Telegram.

Los alumnos valoran positivamente el empleo de canales que usan en su día a día y las lecciones de pequeña duración en intensidad. 
A comparative analysis of face to face instruction vs. Telegram mobile instruction in terms of narrative writing (Heidari et al., 2018).
Estudia la efectividad de Telegram en un curso de inglés de certificación del TOEFL aplicando una metodología MALL.
El aprendizaje a través de Telegram ayudó a los alumnos a sacar mejor nota en el examen de inglés.

Nota: ESP (english for specific purposes), TOEFL (test of english as a foreign language) y MALL (mobile assisted language learning).

Fuente: elaboración propia.

\section{Marco teórico}

El marco teórico se articula sobre dos ejes: los modelos de aceptación de la tecnología móvil, en concreto el modelo TAM, diseñado por Davis (1985), y las teorías sobre la usabilidad pedagógica. Para nuestro estudio se han adaptado y ampliado los 10 criterios de evaluación de la usabilidad pedagógica de Nokelainen (2004).

\subsection{Modelo TAM}

El modelo TAM es uno de los modelos más utilizados y presentes en la literatura científica a la hora de analizar los condicionantes actitudinales y motivacionales de los usuarios en relación con el uso de tecnología, páginas web o aplicaciones móviles.

El marco teórico se detalla gráficamente en la figura 1, que tiene como objetivo explicar la ruta lógica que se va a seguir para explicar dicho marco. En primer lugar, se estudian los principales modelos de aceptación de la tecnología que

El modelo TAM es una de las teorías sobre la adaptación de las tecnologías más utilizadas y presentes en la literatura científica a la hora de analizar los condicionantes actitudinales y motivacionales de los usuarios en relación con el uso de tecnología, páginas web o aplicaciones móviles existen. Se selecciona TAM como teoría de aceptación por el amplio espectro de los estudios realizados y se define el constructo que se va a estudiar y la facilidad de uso o usabilidad. De entre todos los modelos sobre usabilidad pedagógica que se estudian se selecciona el de Nokelainen, como se verá en mayor profundidad en el apartado 3.2. Por último, se analizan los dos atributos más importantes, que son la usabilidad técnica y la usabilidad pedagógica. 
Figura 1. Marco teórico

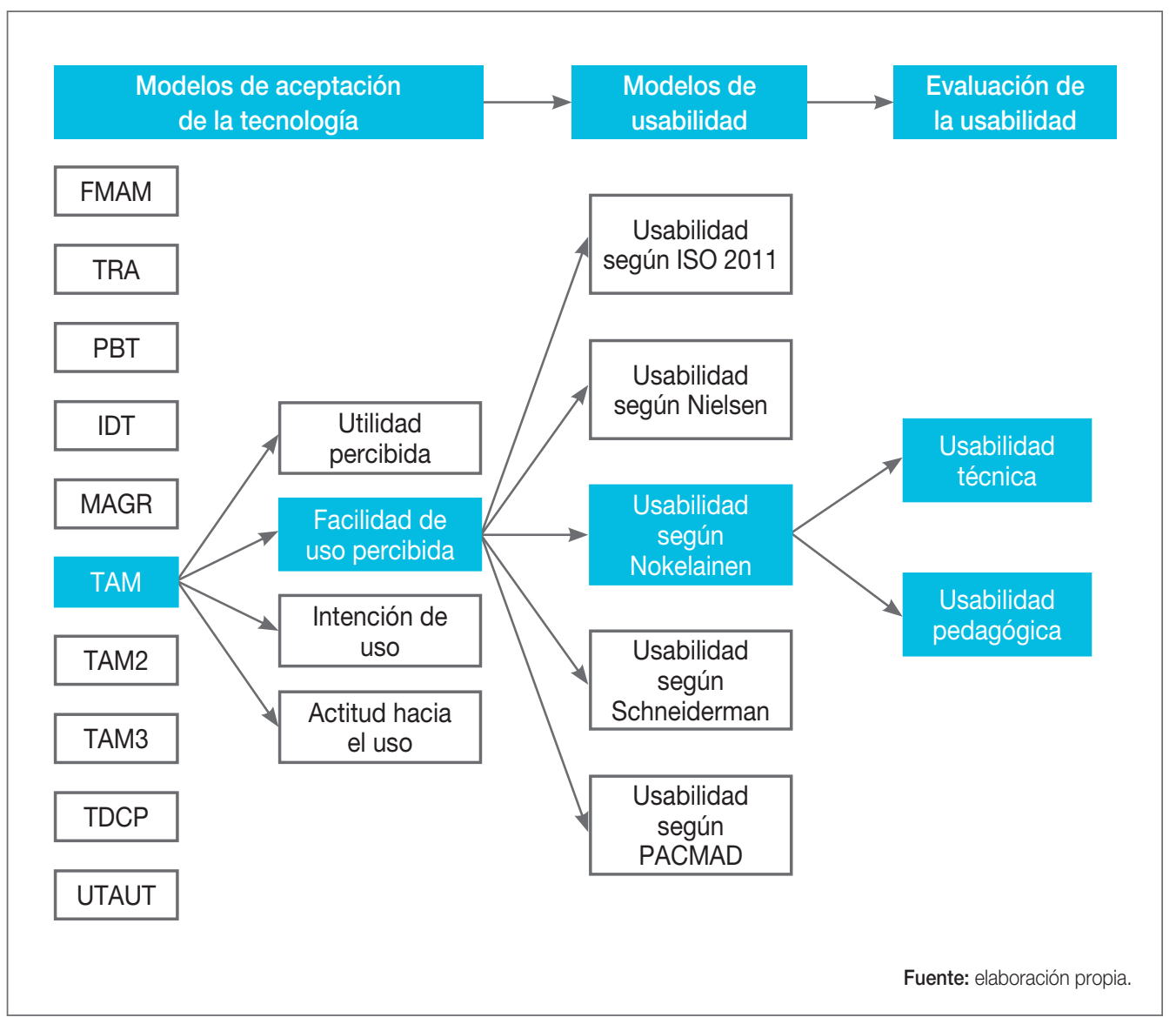

\subsection{Modelos de usabilidad pedagógica}

Se pueden comparar los distintos modelos de usabilidad pedagógica -International Standards Organization (ISO) ${ }^{5}$, Nielsen, Shneiderman y PACMAD- y relacionar sus atributos con los modelos de aprendizaje para analizar esa perspectiva desde el punto de vista de la usabilidad pedagógica. En el caso de nuestro estudio se han seleccionado los trabajos de Nokelainen, que introduce 10 criterios de usabilidad pedagógica. En la figura 2 se puede ver el cuadro en el que se recogen los constructos propios de cada teoría.

\footnotetext{
5 Norma ISO 25010 (2011).
} 
Figura 2. Comparación de los modelos de usabilidad

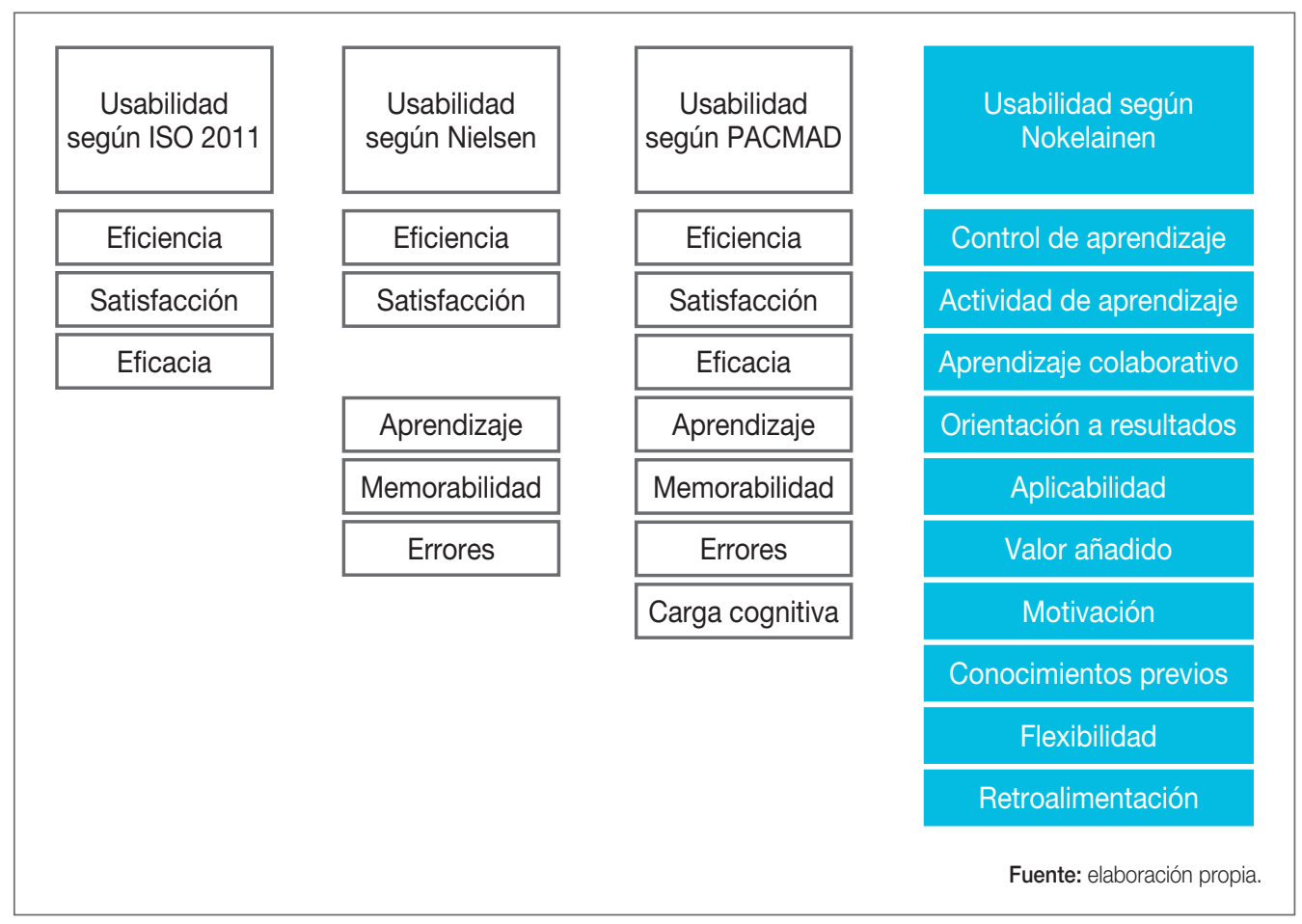

El análisis de la usabilidad pedagógica no debería limitarse a aquellos atributos que hacen que la aplicación funcione o que sea accesible al alumno. Hay que analizarla desde un punto de vista heurístico, valorando aspectos tales como la evaluación del aprendizaje, el nivel de mejora del alumno, la capacidad para incorporar conocimiento, la forma en la que se comunican docente y alumno o, en definitiva, la experiencia de usuario.

\begin{abstract}
La usabilidad pedagógica hay que analizarla desde un punto de vista heurístico, valorando aspectos como la evaluación del aprendizaje, el nivel de mejora del alumno, la capacidad para incorporar conocimiento [...] o, en definitiva, la experiencia de usuario
\end{abstract}

\subsection{Estudio del caso: población, muestra y diseño del cuestionario}

\section{A) Población y muestra}

La población total del caso de estudio asciende a 1.205 alumnos que han realizado el curso de finanzas personales durante los 14 meses que duró el experimento. La muestra 
de nuestro estudio asciende a 339 alumnos, es decir, la tasa de respuesta de la encuesta fue de un $28,13 \%$. En relación con el género, el 8,30\% son mujeres (28 alumnas), con una edad media de 42,03 años. El 91,70\% son hombres (311 alumnos), con una edad media de 38,69 años. Este dato condiciona el análisis de sesgo de género.

La edad media total asciende a 38,97 años, siendo el más joven un alumno con 18 años y el mayor con 72 años. El rango de edad se sitúa en el $22 \%$, para menores de 30 años; en el $34 \%$, entre 30 y 40 años; en el $34 \%$, para mayores de 40 años; y en el $11 \%$, para mayores de 50 años. La moda se establece en 38 y 39 años, con 17 alumnos cada franja de edad.

En relación con la fecha en la que se hizo la encuesta, el 54,90 \% había empezado el curso hacía menos de 6 meses; el 32,20\%, entre 6 meses y 1 año; y el 13\%, hacía más de 1 año.

\section{B) Diseño del cuestionario}

Para el envío de los cuestionarios a los alumnos y la recogida de los datos se han utilizado dos tipos de herramientas: una aplicación externa para el diseño y la elaboración del cuestionario (Google Forms) y el propio canal de Telegram para el envío de la encuesta y la recogida de datos.

El cuestionario está dividido en cuatro bloques:

- Primer bloque. Incorpora los datos e información sociocultural del alumno.

- Segundo bloque. Se adapta el cuestionario system usability scale (SUS) para el análisis de la usabilidad tecnológica.

- Tercer bloque. Se adapta el cuestionario de aprendizaje pedagógicamente significativo (pedagogically meaningful learning questionnaire [PMLQ]) para el análisis de la usabilidad pedagógica.

- Cuarto bloque. En este apartado el alumnado puede añadir sus opiniones personales.

Aunque el estudio se centra en la usabilidad pedagógica, es imprescindible analizar antes si Telegram cumple con los mínimos exigibles en términos de usabilidad técnica. Es de suponer que Telegram, dado su origen, funcionalidades y número de usuarios, goce de altos estándares de usabilidad, pero lo correcto es hacer este análisis.

Para ello se ha elegido el cuestionario SUS por su sencillez, eficiencia y por ser el que mejores resultados obtiene independientemente del tamaño de la muestra, como se desprende del estudio de Tullis y Stetson (2004). 
El cuestionario consta de 10 preguntas que consisten en una afirmación sobre aspectos relativos a la usabilidad técnica de un sistema, sitio web o aplicación, según convenga. Además, se aporta una calificación en una escala de cinco puntos que oscila de «Muy en desacuerdo» (puntúa 1) a «Muy de acuerdo» (puntúa 5). El sistema de puntuación viene definido por una escala de estilo Likert (en nuestro estudio, del 1 al 5). Después de una serie de cálculos, se genera un resultado numérico de dos cifras con dos decimales que se debe relacionar dentro de una escala.

El cálculo se realiza tomando los resultados de las encuestas de las posiciones impares 1. ${ }^{a}, 3 .^{a}, 5 .^{a}, 7 .^{a}$ y $9 .^{a}$. A estas posiciones, se les resta 1 punto. A continuación, se le resta al número 5 el resultado de las posiciones pares $2 .^{a}, 4 .^{a}, 6 .^{a}, 8 .^{a}$ y $10 .^{a}$. Una vez hechas estas operaciones aritméticas, se multiplica la suma de los resultados por 2,5 y se obtiene un valor global de la escala. El resultado final se situará entre un rango de 0 y 100 , teniendo en cuenta que a partir de 68 puntos el resultado de la usabilidad técnica de la aplicación es altamente positiva. Los 10 puntos seleccionados aglutinan las respuestas más explicativas para analizar la usabilidad técnica y, además, cuentan con una correlación entre 0,7 y 0,9.

En relación con la usabilidad pedagógica se adapta el cuestionario PMLQ. Este cuestionario consta a su vez de dos bloques de preguntas: un primer bloque con 49 preguntas que analizan la usabilidad técnica en relación con la formación y un segundo bloque con 51 preguntas que analizan la usabilidad pedagógica en detalle. En nuestro caso estas 100 preguntas se han completado con otras 30 hasta llegar a un cuestionario final de 130 preguntas.

El propio Nokelainen advierte que muchas de las preguntas estudian aspectos similares o no tienen una diferenciación aparente. Ello es debido a que la «usabilidad pedagógica» es un concepto con una alta carga de subjetividad. Una vez hecha la encuesta, es recomendable agrupar los resultados por criterios homogéneos de usabilidad.

Estos 10 criterios se utilizarán en el apartado 5.1 (A) para realizar el análisis de los resultados. Por tanto, una vez realizada la encuesta se hará un análisis de correlaciones y se agruparán los resultados por criterios de usabilidad y, a su vez, según las proposiciones.

\subsection{Descripción y contenido del curso de finanzas personales}

\section{A) La metodología del curso}

El diseño del curso se ha planteado bajo las siguientes premisas: sencillez del método de aprendizaje, material didáctico actualizado a diario, combinación de metodologías, canal móvil como eje del curso y especial importancia del aprendizaje por competencias.

El curso de finanzas personales nace con una vocación totalmente online. Los alumnos se dan de alta en el canal de Telegram y es a través de la propia aplicación donde 
reciben la formación. Por tanto, el canal de Telegram se convierte en la única infraestructura (o aula) donde los alumnos reciben los contenidos del curso, como vídeos, audios, cuestionarios, feedback a su trabajo o resolución de dudas. Telegram sirve, además, de soporte y repositorio de documentación e información, al tiempo que se convierte en un aula de prácticas o laboratorio virtual desde donde poder relacionarse con los alumnos o repasar el contenido teórico.

El curso comienza con un mensaje de bienvenida y con el envío de las primeras comunicaciones en las que se explica nuevamente el programa y los objetivos del curso. Además, se envía una play list con cerca de 200 vídeos con la teoría (aproximadamente 7 horas de material) y los contenidos que hay que estudiar. Estos contenidos han sido elaborados ad hoc para este curso. Cabe señalar que el curso tiene una metodología que permite incorporar alumnos en cualquier momento. Esta flexibilidad es posible porque Telegram posibilita al nuevo alumno ver la información publicada en el canal con anterioridad a su incorporación al grupo.

Respecto a los horarios, se establecen en función de cada canal. El aula móvil a través de Telegram permanece activa durante todo el día, puesto que no existe una planificación o sistematización de los mensajes. Los mensajes se envían cuando lo decide el equipo docente y es el alumno quien escoge el momento de conectarse o de leer el mensaje y su contenido. En relación con la dedicación, esta depende de muchos factores, como son el perfil del alumno, sus conocimientos previos, su tiempo disponible y, en definitiva, el interés que demuestre. El método, la infraestructura del aula y la orientación de los docentes se han diseñado para reducir los inconvenientes de la falta de tiempo de dedicación, la falta de conocimientos previos o la disponibilidad horaria y física.

El método de aprendizaje seguido está inspirado en los modelos de PBL. Los fundamentos del modelo PBL se remontan a John Dewey (1963), profesor de la Universidad de Chicago, que junto con otros docentes empezaron a investigar este modelo. La primera experiencia fue realizada por Noor y Hussin (1970). Esta idea básica se perfecciona por Vygotsky (1978), quien incorpora la idea del docente como mediador o guía dentro del programa de aprendizaje.

Los tres principales atributos que definen el modelo PBL son la relación entre el docente y los alumnos, la relación entre el docente y los contenidos y la relación entre los alumnos y los contenidos. 


\section{B) Los objetivos y las competencias del curso de finanzas personales}

El objetivo principal del curso es que los alumnos se inicien en el estudio de las finanzas personales y en los mercados financieros. Es importante que los alumnos aprendan a gestionar por sí mismos sus inversiones de una manera eficiente y que tengan la posibilidad y la capacidad de seguir avanzando en el conocimiento de los mercados financieros en general.

Las competencias generales se pueden resumir en capacidad para comprender, analizar y sintetizar las diferentes situaciones económicas, capacidad para la toma de decisiones sobre sus finanzas y habilidad para la búsqueda de información. Las competencias específicas se concretan en la capacidad para entender el funcionamiento de los mercados financieros, capacidad para clasificar y medir los riesgos de la inversiones y capacidad para hacer un análisis y selección de inversiones. Las competencias trasversales se refieren a la capacidad para afrontar situaciones estresantes en entornos de presión, tener pensamiento analítico y deductivo y capacidad para volver a aprender conceptos conocidos desde otra perspectiva.

\section{C) La importancia del equipo docente}

Los docentes (también llamados «tutores») desempeñan un papel destacado en la actividad de aprendizaje. Son ellos quienes elaboran los materiales de enseñanza, delimitan los objetivos de aprendizaje de conformidad a cierto mapa de conocimiento y son el «motor de arranque» del curso.

Cada día el docente incorpora contenidos nuevos. Esto permite enviar series de vídeos diariamente sobre diferentes aspectos relativos a las finanzas personales. Otro de los contenidos que el docente incorpora diariamente en el canal son los audios sobre cuestiones que hay que explicar o capturas de pantallas con la actividad realizada por los alumnos.

Las tutorías se realizan individualmente a través de Telegram, WhatsApp, correo electrónico o incluso redes sociales, pero fuera del canal principal del curso. En este espacio, el alumno es libre de preguntar lo que necesite y son los docentes quienes siempre están disponibles para atender las dudas.

El sistema de evaluación y el seguimiento continuo se hacen a través de los cuestionarios que pueden desarrollarse por medio del propio sistema de encuestas de Telegram o de enlaces externos a otras plataformas.

\section{D) El alumno como protagonista}

La participación de los alumnos es muy importante en este curso. Los auténticos dinamizadores y conductores del curso son los docentes, pero los alumnos van matizando con su trabajo y desempeño los contenidos e intensidad del curso. 
Uno de los aspectos más importantes en relación con los alumnos es que son generadores de contenidos. A través de sus dudas, sus experiencias como inversores, sus casos personales o la propia dinámica del curso van generando contenidos que el docente tendrá que gestionar y compartir con el resto de alumnos.

Los alumnos van aprendiendo por ellos mismos a través de sus propias experiencias y las de otros compañeros. En muchas ocasiones no hay una sola respuesta correcta y las interpretaciones ofrecidas por la «comunidad» hacen el aprendizaje enriquecedor y permanente. Por último, es importante destacar que los alumnos aprenden haciendo y experimentando con casos y entornos reales.

\section{E) La importancia de los contenidos y los materiales}

Los contenidos compartidos a través del canal Telegram son de diferentes tipos: vídeos, fotos, archivos, enlaces y audios, que ascienden a 18.494 contenidos. Las estadísticas de los contenidos incorporados al canal fueron las siguientes: vídeos y fotos (17.567), archivos (26), enlaces (383), audios (9) y mensajes de voz (509).

El vídeo es la base de la estrategia de contenidos del curso. Ello es debido a cinco factores: el alto grado de visualización, la personalización de los contenidos, ser un facilitador del aprendizaje, ofrecer la posibilidad de repetición y ser el formato más consumido por tipo de alumno y dispositivo. En el curso de finanzas personales se enviaron 17.567 vídeos y fotografías. Los vídeos representan un $62,70 \%$ sobre el total de los contenidos del curso.

\author{
El vídeo es la base de la estrategia \\ de contenidos del curso. Ello \\ es debido a cinco factores: \\ el alto grado de visualización, \\ la personalización de los \\ contenidos, ser un facilitador \\ del aprendizaje, ofrecer la \\ posibilidad de repetición y ser \\ el formato más consumido por \\ tipo de alumno y dispositivo. \\ En el curso de finanzas \\ personales se enviaron 17.567 \\ vídeos y fotografías. Los vídeos \\ representan un $62,70 \%$ sobre el \\ total de los contenidos del curso
}

Las fotografías e imágenes son un complemento perfecto a los vídeos, tanto si están producidos con antelación como si son elaborados por el docente durante sus explicaciones de forma personalizada e inmediata. Las fotografías representan un 32,29\% sobre el total de los contenidos del curso.

Los archivos y los enlaces son un buen material complementario, ya que en muchas ocasiones el docente encuentra material interesante de otros profesores o material que se publica en redes sociales profesionales, o bien material que los alumnos le aportan y que él considera como relevante. Este material es compartido en forma de archivo, habitualmente en formato PDF, que es el que ofrece más posibilidad de visualización desde cualquier dispositivo o versión. En el curso de finanzas personales se enviaron 409 archivos y enlaces, que representan un $2,21 \%$ sobre el total de los contenidos del curso. 
En relación con los audios, son un elemento importante que suele emplearse para corregir algún concepto general o errores individuales que conviene compartir con el resto de alumnos. Por tanto, el alumno sabe que los audios obedecen a alguna situación importante que debe corregirse. En el curso de finanzas personales se enviaron 518 archivos, que representan un $2,80 \%$ sobre el total de los contenidos del curso.

\section{Resultados de la investigación}

El objetivo del presente apartado es mostrar los resultados cuantitativos y cualitativos recogidos en la encuesta. Se ha utilizado la aplicación Google Forms que procesa automáticamente los informes, aportando datos y gráficos en formato Excel. El archivo se exporta y se trata con SPSS para el análisis básico, y los datos se filtran a través de tablas dinámicas de Excel.

\subsection{Resultados cuantitativos}

\section{A) Resultado usabilidad técnica según SUS}

Como ya se explicó en el apartado 3.3 (B), y como puede verse en el cuadro 3, el cálculo se realiza tomando los resultados de las encuestas de las posiciones impares $1 .^{\mathrm{a}}, 3 .^{\mathrm{a}}, 5 .^{\mathrm{a}}$, 7. ${ }^{\mathrm{a}}$ y $9 .^{\mathrm{a}}$, y a estas posiciones se les resta 1 punto. A continuación, se le resta al número 5 el resultado de las posiciones pares $2 .^{a}, 4 .^{a}, 6 .^{a}, 8 .^{a}$ y $10 .^{a}$.

Cuadro 3. Cálculo del índice de SUS

\begin{tabular}{l|c|c|c|c|c|c|c|c|c|c|c|c} 
& $\mathbf{S 1}$ & $\mathbf{S 2}$ & $\mathbf{S 3}$ & $\mathbf{S 4}$ & $\mathbf{S 5}$ & $\mathbf{S 6}$ & $\mathbf{S 7}$ & $\mathbf{S 8}$ & $\mathbf{S 9}$ & $\mathbf{S 1 0}$ & $\begin{array}{c}\text { Media } \\
\text { total }\end{array}$ & SUS \\
\hline Media & 3,74 & 1,35 & 4,53 & 1,32 & 4,06 & 1,50 & 4,41 & 1,41 & 4,12 & 1,26 & - & - \\
\hline Cálculo & 4,74 & 3,65 & 3,53 & 3,68 & 3,06 & 3,50 & 3,41 & 3,59 & 3,12 & 3,74 & 36 & 90 \\
\hline Aplicación & -1 & $5-$ & -1 & $5-$ & -1 & $5-$ & -1 & $5-$ & -1 & $5-$ & 2,5 & 68 \\
\hline
\end{tabular}

Fuente: elaboración propia.

La nota final que ha tenido el estudio de la usabilidad técnica de Telegram asciende a 90, mejorando en un $36 \%$ el mínimo exigido por SUS de 68. Por tanto, se puede afirmar que Telegram tiene una alta usabilidad técnica. 
B) Resultados sobre la adecuación de Telegram como herramienta para el mobile learning en relación con los modelos de aprendizaje

La puntuación media obtenida a la pregunta relacionada con la proposición 1 resulta de 8,67 , siendo la nota más frecuente el 10, con un 30,70\% (104 alumnos); el 8, con un $28 \%$ (95 alumnos); y el 9, con un 27,70\% (94 alumnos). El porcentaje menor que 5 asciende a $0,60 \%$, con 2 alumnos.

C) Resultados sobre la adecuación de Telegram como herramienta para el mobile learning que complementa la labor del docente, pero no la sustituye

La puntuación media obtenida a la pregunta relacionada con la proposición 1 resulta de 8,66 , siendo la nota más frecuente el 10, con un 36,20\% (122 alumnos); el 8, con un $25,20 \%$ (87 alumnos); y el 9, con una $23,40 \%$ (79 alumnos). El porcentaje menor que 5 asciende a $1,80 \%$, con 6 alumnos.

D) Resultados sobre la adecuación de Telegram como herramienta para el mobile learning que facilita la interrelación entre los alumnos y les permite avanzar en el aprendizaje compartiendo experiencias, conocimientos y resultados

La puntuación media obtenida a la pregunta relacionada con la proposición 1 resulta de 8,20, siendo la nota más frecuente el 10 , con un $34,80 \%$ (118 alumnos); el 8, con un 21,20\% (72 alumnos); y el 9 , con una $19,20 \%$ (65 alumnos). El porcentaje menor que 5 asciende a 5,70\%, con 19 alumnos.

Los alumnos destacan: «Me ha sorprendido positivamente Telegram. Me ayuda en mi aprendizaje, a repasar la teoría y a guardar los vídeos con explicaciones»

E) Resultados sobre la adecuación de Telegram como herramienta para el mobile learning que alcanza el máximo valor pedagógico cuando se incorporan contenidos audiovisuales

La puntuación media obtenida a la pregunta relacionada con la proposición 1 resulta de 9,23 , siendo la nota más frecuente el 10, con un 55,50\% (188 alumnos); el 9, con un $23,30 \%$ (79 alumnos); y el 8, con una 14,70\% (50 alumnos). El porcentaje menor que 5 asciende a $0,60 \%$, con 2 alumnos. 
F) Resultados sobre la adecuación de Telegram como herramienta para el mobile learning que alcanza el máximo valor pedagógico cuando compagina diferentes dispositivos móviles (teléfono móvil y tablet o teléfono móvil y portátil o PC)

La puntuación media obtenida a la pregunta relacionada con la proposición 1 resulta de 8,57 , siendo la nota más frecuente el 10, con un 37,20\% (126 alumnos); el 9, con un $25,10 \%$ (85 alumnos); y el 8, con un 18,60 \% (63 alumnos). El porcentaje menor que 5 asciende a $1,80 \%$, con 6 alumnos.

\subsection{Resultados cualitativos}

Como se indicó en el apartado $3.3(\mathrm{~B})$, el cuarto bloque de la encuesta hace referencia a la última pregunta del formulario, un espacio en el que se les da a los alumnos la oportunidad de comentar aquellos aspectos que más les han gustado o que deben ser revisados.

Los resultados han ascendido a un total de 811 opiniones que son de gran valor porque complementan los resultados cuantitativos. Se aporta una pequeña selección en el apartado $5.2(B)$.

\section{Análisis de resultados}

\subsection{Análisis cuantitativo}

\section{A) Estadísticos simples}

Se han calculado los estadísticos simples y se han ordenado los resultados medios de tal forma que la P4 resulta ser la mejor valorada, con un 9,23 de nota. En segundo lugar, la $\mathrm{P} 1$, con un resultado de 8,67, casi a la par que la P2, con un 8,66. En cuarto lugar, la P5, con un 8,57, y, por último, la peor valorada es la P3, con un 8,20 (véase cuadro 4). La valoración media de los resultados parciales de las cinco proposiciones es de 8,66, quedando por encima o igualando este resultado medio la P4, la P1 y la P2, y quedando por debajo la P5 y P3.

En relación con la desviación sobre la media, vemos que la P3 presenta una dispersión mayor que en el resto, con un 2,07. Ello indica la dispersión en los resultados que se confirmará a lo largo del análisis de este apartado y del siguiente. El resto de proposiciones se ordena de la siguiente forma: P5, con una desviación estándar de 1,71, que indica 
también una alta dispersión en los resultados; P2, con 1,50; P1, con 1,27; y, por último, la $\mathrm{P} 4$, con 1,13, como puede verse en el cuadro 4.

\section{Cuadro 4. Resumen de los estadísticos simples}
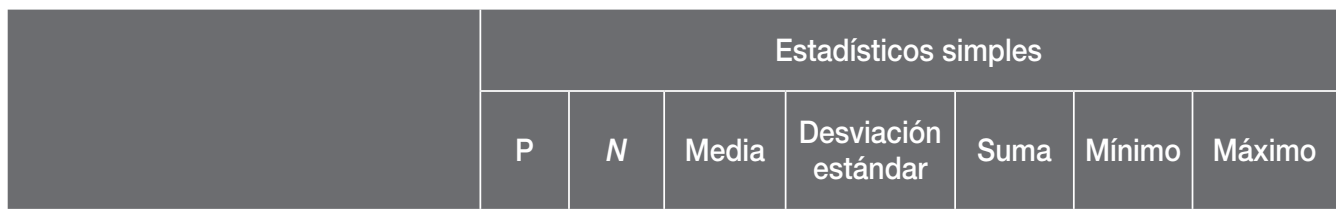

Telegram es una herramienta adecuada para el mobile learning desde el punto de vista de la usabilidad,

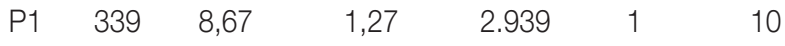
tanto técnica como pedagógica.

Telegram, como herramienta de mobile learning, no sustituye la labor de los docentes, sino que la comple$\mathrm{P}$ P2 339 8,66 1,50 2.937 10 menta.

Telegram, como herramienta de mobile learning, facilita la interrelación entre los alumnos y les permite avanzar en el aprendizaje, compartiendo tados. experiencias, conocimientos y resul-
P
339
8,20
2,07
2.781
10

Telegram, como herramienta de mobile learning, alcanza su máxima usabilidad pedagógica cuando se incorpoP4

P4 339

9,23

1,13

3.128

1

10 ran contenidos audiovisuales.
Telegram, como herramienta de mobile learning, alcanza su máxima usabilidad pedagógica cuando compagina diferentes dispositivos móviles (teléfono móvil y tablet o teléfono móvil y portátil o $\mathrm{PC})$.

$\begin{array}{lllllll}\text { P5 } & 339 & 8,57 & 1,71 & 2.905 & 1 & 10\end{array}$

Fuente: elaboración propia.

Para contar con un análisis más explicativo que el de los resultados medios se analizan los percentiles, siendo también la P4 la que tiene la máxima nota desde el percentil 50 hasta el percentil 100. Llama la atención que solo se obtiene una puntuación menor que 5 en el percentil 0 de todas las proposiciones. 


\section{B) Matriz del gráfico de dispersión}

A la hora de representar gráficamente la dispersión, es interesante comprobar que los resultados de las proposiciones se aglutinan en las colas situadas en la derecha (de mayor puntuación) y que las barras que dibujan tienen sentido ascendente, como puede verse en la figura 3.

Figura 3. Matriz del gráfico de dispersión

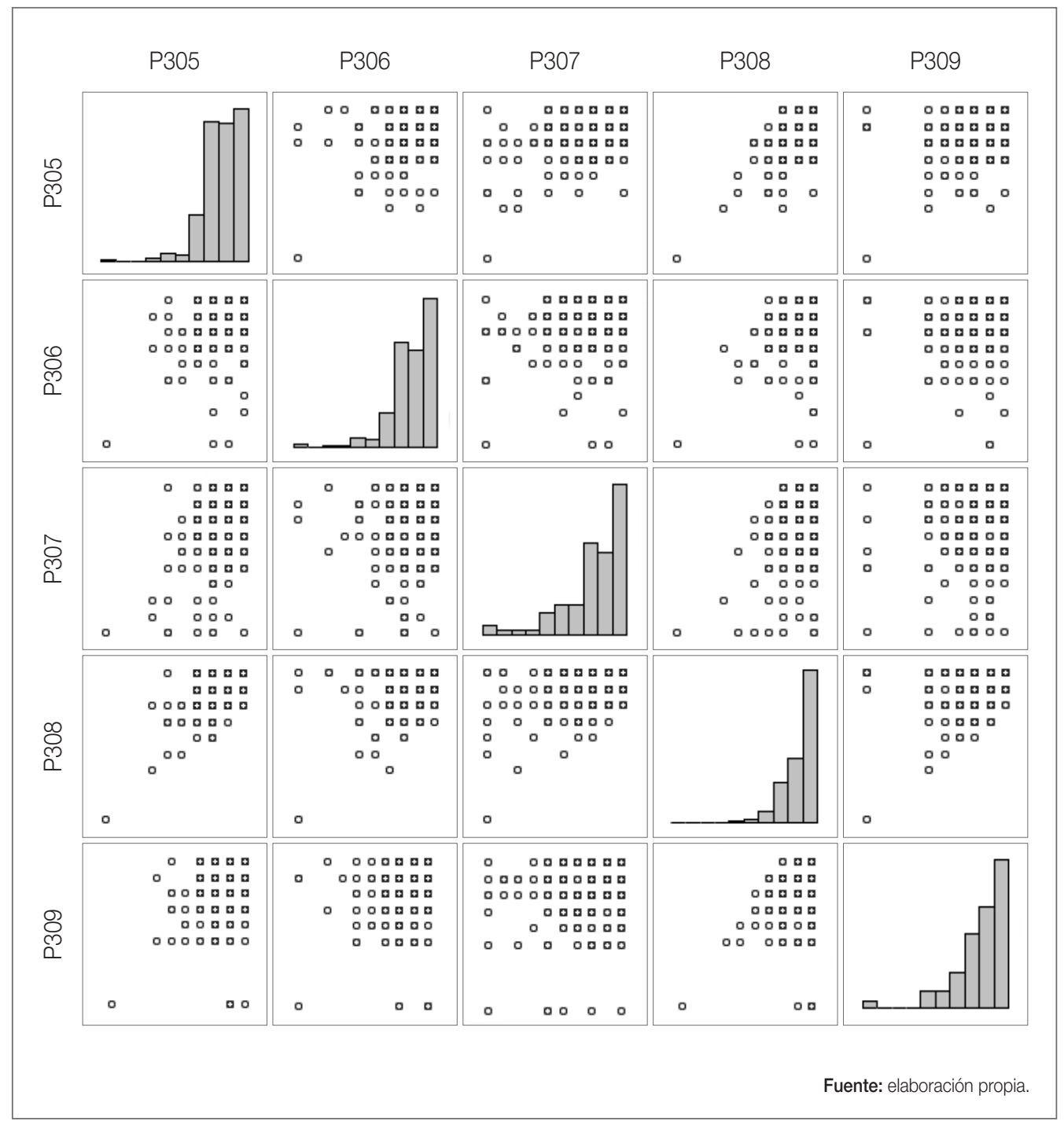




\section{C) Matriz de correlación}

Se han realizado los cálculos pertinentes para calcular la matriz de correlaciones o, como se suele utilizar en la literatura científica, los coeficientes de correlación de Pearson para una muestra de $N$ (339), suponiendo probabilidad (Prob.) $>0, H 0$ y Rho $=0$, como se puede ver en el cuadro 5 .

Cuadro 5. Cálculo del coeficiente de correlación de Pearson para una muestra de $N$ (339), suponiendo probabilidad (Prob.) $>0, H O$ y $\mathrm{Rho}=0$

\begin{tabular}{|c|c|c|c|c|c|}
\hline \multirow{4}{*}{$\mathbf{P}$} & \multicolumn{5}{|c|}{ Matriz de correlación } \\
\hline & \multicolumn{5}{|c|}{ Coeficientes de correlación de Pearson ( $N=339$ ) } \\
\hline & \multicolumn{5}{|c|}{ Prob. $>|r|$, suponiendo $\mathrm{HO}$ y $\mathrm{Rho}=0$} \\
\hline & P1 & P2 & P3 & P4 & P5 \\
\hline \multirow{2}{*}{ P1 } & 1 & 0,43144 & 0,48399 & 0,62839 & 0,33201 \\
\hline & & $<0,0001$ & $<0,0001$ & $<0,0001$ & $<0,0001$ \\
\hline \multirow{2}{*}{ P2 } & 0,43144 & 1 & 0,30651 & 0,42736 & 0,29214 \\
\hline & $<0,0001$ & & $<0,0001$ & $<0,0001$ & $<0,0001$ \\
\hline \multirow{2}{*}{ P3 } & 0,48399 & 0,30651 & 1 & 0,46632 & 0,30319 \\
\hline & $<0,0001$ & $<0,0001$ & & $<0,0001$ & $<0,0001$ \\
\hline \multirow{2}{*}{ P4 } & 0,62839 & 0,42736 & 0,46632 & 1 & 0,42632 \\
\hline & $<0,0001$ & $<0,0001$ & $<0,0001$ & & $<0,0001$ \\
\hline \multirow{2}{*}{ P5 } & 0,33201 & 0,29214 & 0,30319 & 0,42632 & 1 \\
\hline & $<0,0001$ & $<0,0001$ & $<0,0001$ & $<0,0001$ & \\
\hline
\end{tabular}

Para mayor claridad se han ordenado y convertido los cálculos y se les han asignado unos colores más oscuros a aquellas respuestas-proposiciones que ofrecían una mayor 
correlación entre sí. Los resultados se pueden analizar en el cuadro 6. Se observa que las proposiciones que mayor correlación tienen son P4 y P1, lo que indica que los contenidos audiovisuales influyen de una manera muy importante, con un $63 \%$ de coeficiente sobre las metodologías de aprendizaje.

Además, las proposiciones que mantienen una relación positiva y alta son P3 y P1, siendo importante la relación que los alumnos muestran interactuando y su impacto positivo sobre la metodología de aprendizaje, con un $48 \%$. Muy seguidas, con un $47 \%$, se sitúan las correlaciones entre P3 y P4. Otras proposiciones que también mantienen una alta y positiva correlación, con un $43 \%$, son la P2 con la P1 y con la P4, siendo muy alta la influencia de los docentes en la metodología y en la elaboración de los contenidos. Aun manteniendo todas las proposiciones una correlación positiva, se observa que la menor correlación la presentan la P5 con la P2, con un 29 \%, siguiendo la lógica de que la labor de los docentes tiene relación con los dispositivos, pero no determina de forma concluyente los resultados de esta proposición.

Cuadro 6. Matriz de correlaciones

\begin{tabular}{|c|c|c|c|c|c|c|}
\hline Matriz de correlaciones & $P$ & P1 & $\mathrm{P} 2$ & P3 & P4 & P5 \\
\hline $\begin{array}{l}\text { Telegram es una herramienta adecuada para el } \\
\text { mobile learning desde el punto de vista de la usa- } \\
\text { bilidad, tanto técnica como pedagógica. }\end{array}$ & P1 & $100 \%$ & $43 \%$ & $48 \%$ & $63 \%$ & $33 \%$ \\
\hline $\begin{array}{l}\text { Telegram, como herramienta de mobile learning, } \\
\text { no sustituye la labor de los profesores, sino que } \\
\text { la complementa. }\end{array}$ & $\mathrm{P} 2$ & $43 \%$ & $100 \%$ & $31 \%$ & $43 \%$ & $29 \%$ \\
\hline $\begin{array}{l}\text { Telegram, como herramienta de mobile learning, } \\
\text { facilita la interrelación entre los alumnos y les per- } \\
\text { mite avanzar en el aprendizaje, compartiendo ex- } \\
\text { periencias, conocimientos y resultados. }\end{array}$ & P3 & $48 \%$ & $31 \%$ & $100 \%$ & $47 \%$ & $30 \%$ \\
\hline $\begin{array}{l}\text { Telegram, como herramienta de mobile learning, } \\
\text { alcanza su máxima usabilidad pedagógica cuan- } \\
\text { do se incorporan contenidos audiovisuales. }\end{array}$ & P4 & $63 \%$ & $43 \%$ & $47 \%$ & $100 \%$ & $43 \%$ \\
\hline $\begin{array}{l}\text { Telegram, como herramienta de mobile learning, } \\
\text { alcanza su máxima usabilidad pedagógica cuando } \\
\text { compagina diferentes dispositivos móviles (teléfono } \\
\text { móvil y tablet o teléfono móvil y portátil o PC). }\end{array}$ & P5 & $33 \%$ & $29 \%$ & $30 \%$ & $43 \%$ & $100 \%$ \\
\hline
\end{tabular}




\subsection{Análisis cualitativo}

\section{A) Análisis 10 criterios de Nokelainen}

Se ha utilizado un criterio sencillo, consignando en cada pregunta «si se cumplen» o «no se cumplen» los criterios en, al menos, un $50 \%$ o si la nota supera el 5 de media. El mero hecho de ordenar las respuestas según los criterios y darles una sencilla valoración aporta mucha información de cara a las conclusiones finales, como se puede ver en el cuadro 7.

\section{Cuadro 7. Análisis 10 criterios de Nokelainen}

\begin{tabular}{|c|c|c|c|c|}
\hline $\mathbf{P}$ & Criterio & Bloque & Pregunta & Resultado \\
\hline \multirow{11}{*}{1} & \multirow{7}{*}{ Valor añadido } & \multirow{5}{*}{0} & $\begin{array}{l}0.11 \text { ¿Harías más cursos de esta u otras materias a } \\
\text { través de Telegram? }\end{array}$ & Sí \\
\hline & & & $\begin{array}{l}0.15 \text {. Valoración de Telegram como herramienta para } \\
\text { aprender finanzas personales. }\end{array}$ & Sí \\
\hline & & & $\begin{array}{l}0.17 \text {. Un factor importante del uso de Telegram es que } \\
\text { es gratis. }\end{array}$ & Sí \\
\hline & & & $\begin{array}{l}\text { 0.19. Me gusta contarle a mis amigos y compañeros que } \\
\text { estoy haciendo un curso a través de Telegram. }\end{array}$ & No \\
\hline & & & 0.20. Telegram ha cambiado mi forma de aprender. & Sí \\
\hline & & & 2.1. Telegram es una parte importante de mi aprendizaje. & Sí \\
\hline & & 2 & $\begin{array}{l}\text { 2.56. Telegram me permite aprender con mayor rapidez } \\
\text { que con otros sistemas. }\end{array}$ & Sí \\
\hline & \multirow{4}{*}{$\begin{array}{l}\text { Orientación a } \\
\text { resultados }\end{array}$} & \multirow[b]{2}{*}{0} & 0.13. ¿Tienes pensado abandonar el curso? & Sí \\
\hline & & & $\begin{array}{l}0.14 \text {. Valoración del curso de finanzas personales a tra- } \\
\text { vés de Telegram de } 1 \text { a } 10 \text {. }\end{array}$ & Sí \\
\hline & & \multirow{2}{*}{2} & $\begin{array}{l}\text { 2.28. Creo que Telegram me ayudará a mejorar mi co- } \\
\text { nocimiento en finanzas personales. }\end{array}$ & Sí \\
\hline & & & $\begin{array}{l}\text { 2.57. Telegram es recomendable para la formación en } \\
\text { finanzas personales. }\end{array}$ & Sí \\
\hline
\end{tabular}




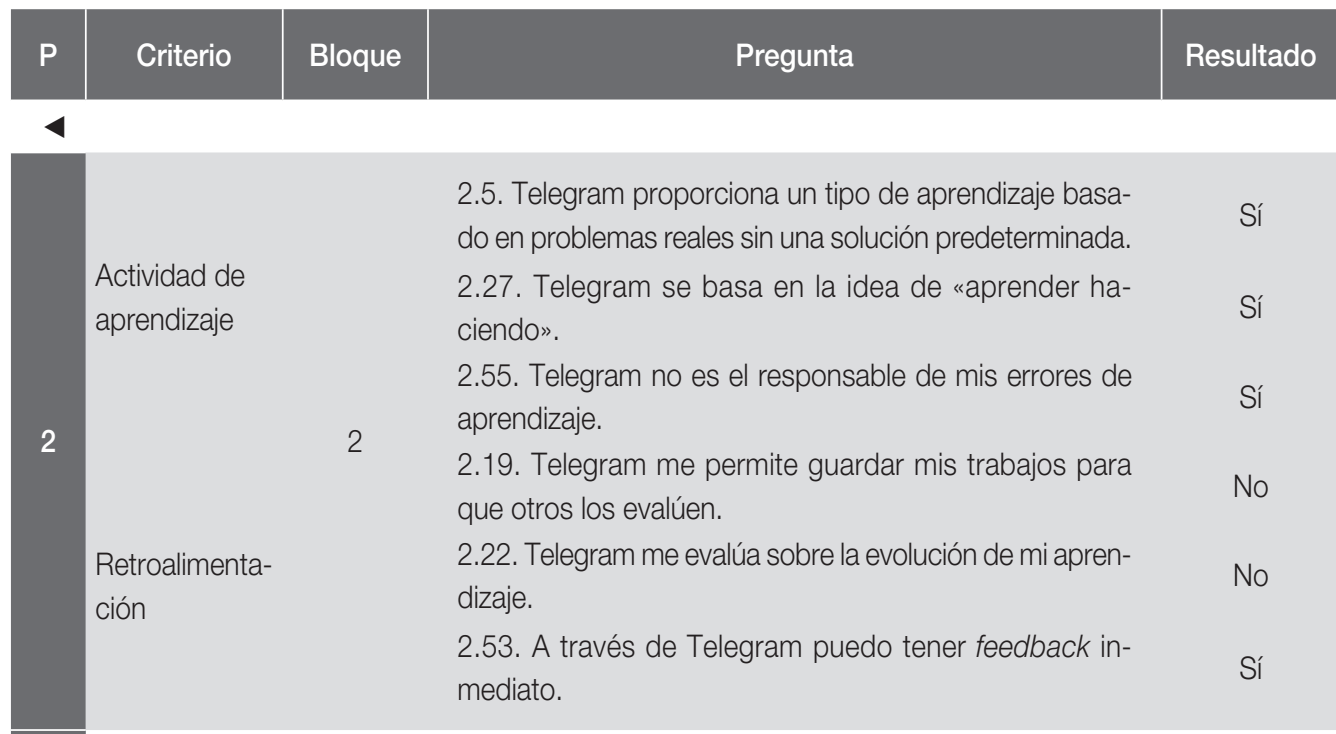

Aprendizaje

0 $\quad$ 0.18. Me gustaría poder interactuar con mis compañeros

colaborativo a través del grupo de Telegram.

2 2.9. Telegram nos permite trabajar en grupo para llegar a nuestras propias soluciones.

2.43. Telegram me obliga a seguir un orden de aprendizaje.

Control de

aprendizaje

3

Motivación

0

Valoración de conocimientos previos

0
2.58. Telegram es una buena opción cuando se trata de adquirir habilidades prácticas.

2.59. Telegram es una buena opción cuando se trata de adquirir conocimientos teóricos.

0.9. ¿Por qué haces el curso de finanzas personales?

0.3. Nivel de estudios (el máximo).

0.4. Ocupación.

0.5. ¿Trabajas o has trabajado en el sector financiero o de gestión de patrimonios?

0.6. Experiencia con Telegram.

0.7. Experiencia haciendo el curso de finanzas personales.

0.8. ¿Cuándo empezaste el curso de finanzas personales?

0.10. ¿Es tu primer curso a través del móvil?
Sí

Sí

Sí

Sí

Sí

Sí

Sí

Sí

Sí 


\begin{tabular}{|c|c|c|c|c|}
\hline $\mathbf{P}$ & Criterio & Bloque & Pregunta & Resultado \\
\hline 4 & & & & \\
\hline \multirow{5}{*}{4} & \multirow{5}{*}{ Aplicabilidad } & \multirow{5}{*}{2} & $\begin{array}{l}\text { 2.32. Recibir imágenes a través de Telegram me ayuda } \\
\text { en mi aprendizaje. }\end{array}$ & Sí \\
\hline & & & $\begin{array}{l}\text { 2.33. Recibir audios a través de Telegram me ayuda en } \\
\text { mi aprendizaje. }\end{array}$ & Sí \\
\hline & & & $\begin{array}{l}\text { 2.34. Recibir gifs animados a través de Telegram me } \\
\text { ayuda en mi aprendizaje. }\end{array}$ & Sí \\
\hline & & & $\begin{array}{l}\text { 2.45. Telegram me permite aprender conceptos nuevos } \\
\text { y repasar los anteriores. }\end{array}$ & Sí \\
\hline & & & $\begin{array}{l}\text { 2.49. Telegram me permite adquirir conocimientos poco } \\
\text { a poco. }\end{array}$ & Sí \\
\hline \multirow{13}{*}{5} & \multirow{13}{*}{ Flexibilidad } & \multirow{12}{*}{1} & 0.12. ¿Echas en falta tener clases presenciales? & Sí \\
\hline & & & $\begin{array}{l}0.16 . \text { ¿Qué dispositivo usas cuando aprendes finanzas } \\
\text { personales a través de Telegram? }\end{array}$ & Sí \\
\hline & & & 1.3. Telegram es fácil de usar. & Sí \\
\hline & & & $\begin{array}{l}\text { 1.7. Telegram funciona sin fallos y nunca se «queda col- } \\
\text { gado». }\end{array}$ & Sí \\
\hline & & & $\begin{array}{l}\text { 1.10. Recuerdo cómo usar Telegram incluso después de } \\
\text { semanas sin utilizarlo. }\end{array}$ & Sí \\
\hline & & & $\begin{array}{l}\text { 1.17. Usar Telegram provoca errores de sistema en mi } \\
\text { móvil. }\end{array}$ & Sí \\
\hline & & & $\begin{array}{l}\text { 1.31. Los colores que usa Telegram en su pantalla se } \\
\text { distinguen bien. }\end{array}$ & Sí \\
\hline & & & $\begin{array}{l}\text { 1.33. Los textos que se aportan a través de Telegram se } \\
\text { ven con claridad. }\end{array}$ & Sí \\
\hline & & & $\begin{array}{l}\text { 1.34. Las imágenes que se ven a través de Telegram se } \\
\text { ven con nitidez. }\end{array}$ & Sí \\
\hline & & & 1.35. El contenido de Telegram carga con rapidez. & Sí \\
\hline & & & 1.36. Telegram funciona sin cortes. & Sí \\
\hline & & & $\begin{array}{l}\text { 1.37. Las imágenes y símbolos que emplea Telegram son } \\
\text { fácilmente reconocibles. }\end{array}$ & Sí \\
\hline & & 2 & $\begin{array}{l}\text { 2.35. Creo que es mejor aprender por Telegram que por } \\
\text { medios convencionales en clase presencial. }\end{array}$ & Sí \\
\hline
\end{tabular}


Como puede verse en el cuadro 8, el concepto de «evaluación» como parte importante del aprendizaje es visto por los alumnos como un factor que hay que mejorar.

Cuadro 8. Otros constructos que no cumplen con los criterios de Nokelainen

\begin{tabular}{|c|c|c|c|c|}
\hline$P$ & Criterio & Bloque & Pregunta & Resultado \\
\hline 1 & Valor añadido & 0 & $\begin{array}{l}\text { 0.19. Me gusta contarle a mis amigos y compañeros que } \\
\text { estoy haciendo un curso a través de Telegram. }\end{array}$ & No \\
\hline \multirow{2}{*}{2} & \multirow{2}{*}{$\begin{array}{l}\text { Retroalimen- } \\
\text { tación }\end{array}$} & \multirow{2}{*}{2} & $\begin{array}{l}\text { 2.19. Telegram me permite guardar mis trabajos para que } \\
\text { otros los evalúen. }\end{array}$ & No \\
\hline & & & $\begin{array}{l}\text { 2.22. Telegram me evalúa sobre la evolución de mi apren- } \\
\text { dizaje. }\end{array}$ & No \\
\hline
\end{tabular}

Fuente: elaboración propia.

Como resumen, se puede afirmar que el análisis de los criterios de Nokelainen en cuanto a la evaluación de la usabilidad pedagógica se cumple en su amplia mayoría.

\section{B) Análisis según los comentarios de los alumnos}

El alto número de respuestas (811) hace imposible incluirlas en el presente documento. Se han seleccionado algunas de las más interesantes únicamente en relación con el método de aprendizaje:

- «La calidad del curso no tiene que ver con Telegram, sino con el contenido y los docentes, pero de cualquier manera considero que es una buena herramienta».

- «Realmente creo que es una gran forma de aprender si no dispones de tiempo para hacer un curso presencial y, aun así, sientes que no solo estás viendo unos vídeos, imágenes, etc., sino que logran transmitir emociones que ayudan».

- «Me ha sorprendido. No pensé que se podía aprender con el móvil».

- «Me ha sorprendido positivamente Telegram porque me ayuda en mi aprendizaje, a repasar la teoría y a guardar los vídeos con las explicaciones».

- «Telegram es un apoyo importante y muy útil a la formación, aunque es un complemento y debe combinarse con otras herramientas». 


\section{Principales hallazgos de la investigación}

En este apartado se ha hecho una síntesis de los principales hallazgos encontrados en el estudio en relación con el valor de Telegram como complemento para el mobile learning.

Las respuestas encontradas a la pregunta de investigación - ¿en qué medida el uso de nuevas herramientas tecnológicas aporta valor a la formación a través de dispositivos móviles?permiten prolongar la discusión hacia otros interrogantes que pueden constituir el punto de partida para nuevas investigaciones.
Este caso de estudio es un ejemplo válido para potenciar Telegram y las nuevas aplicaciones de comunicación para el mobile learning. Estas innovaciones metodológicas y tecnológicas, unidas a los cambios en las motivaciones de docentes y alumnos, presentan una interesante oportunidad de ser estudiadas en profundidad

Este caso de estudio es un ejemplo válido para potenciar Telegram y las nuevas aplicaciones de comunicación para el mobile learning. Estas innovaciones metodológicas y tecnológicas, unidas a los cambios en las motivaciones de docentes y alumnos, presentan una interesante oportunidad de ser estudiadas en profundidad.

Hay que tener en cuenta que la usabilidad no se puede analizar como un término absoluto, sino bajo una serie de particularidades o proposiciones. Por ello, a lo largo del informe, se ha ido construyendo el hilo argumental alrededor de las cinco proposiciones de forma independiente.

Como se ha visto a lo largo de los cinco apartados anteriores, las proposiciones de la investigación hacen referencia a los métodos de aprendizaje, a la importancia de los docentes, a la interrelación entre los alumnos, al valor de los contenidos (en especial, al vídeo) y al uso de los dispositivos. A continuación, se reflexiona sobre los principales hallazgos descubiertos en el presente estudio.

\subsection{El valor pedagógico de Telegram y la metodología de apren- dizaje}

El estudio nos confirma que Telegram es una herramienta válida para el mobile learning.

Cabe recordar que la nota media del curso es de 8,65 y que la tasa de abandono del mismo podría alcanzar un máximo de un 2,90\%.

\author{
El estudio nos confirma que \\ Telegram es una herramienta válida \\ para el mobile learning. La nota \\ media del curso es de 8,65
}




\section{A) Innovación}

Los alumnos ven Telegram como una nueva forma de aprendizaje donde son protagonistas del mismo y construyen su propio itinerario mientras consumen contenidos actualizados, atractivos y útiles de forma ilimitada y a través de cualquier dispositivo. Los alumnos destacan el factor innovación como un conjunto de elementos entre los que se encuentra la sensación de resultar una experiencia enriquecedora, el carácter revolucionario de la aplicación y la utilidad de la misma, que les permite aprender.

\section{B) Omnicanalidad}

Con Telegram se integra el concepto de «omnicanalidad», relacionado con la centralidad en el cliente, la personalización del mismo y la justificación de la multicanalidad como plataforma global, que permite impartir y recibir la formación desde cualquier país del mundo o uso horario.

\section{C) Nueva metodología de aprendizaje}

Telegram se muestra como una metodología en sí misma por medio de la cual se estructura el curso en función de criterios de evaluación de los alumnos a través de cuestionarios propios, trazabilidad del proceso de aprendizaje, ubicuidad, atención activa de los alumnos, retroalimentación inmediata y el vídeo y los contenidos como ejes de la formación. Al mismo tiempo permite que el docente sea generador de contenidos propios y transmisor de las aportaciones de los alumnos, quienes pueden sumarse al curso en cualquier momento.

\subsection{El valor pedagógico de Telegram y los docentes}

La labor de los docentes es fundamental cuando se utiliza la metodología PBL porque ellos son uno de los tres ejes fundamentales (junto con los alumnos y los contenidos) y porque mejoran todos los factores que definen la usabilidad pedagógica.

\author{
La labor de los docentes es \\ fundamental porque ellos son uno \\ de los tres ejes fundamentales y \\ porque mejoran todos los factores
}

\section{A) Los nuevos roles del docente}

Teniendo presente que los docentes han seguido el método de PBL, la función del docente toma un sesgo diferente y se comporta como mentor, tutor, moderador o instructor. 


\section{B) La importancia de las emociones en los docentes}

Los cuatro atributos más relevantes que los alumnos destacan en relación con las emociones en los docentes son la dedicación, la motivación, la cercanía y la interacción. Telegram ayuda al docente a mejorar su propuesta de valor, acercando a los alumnos su conocimiento, experiencia y pasión.

\section{c) La tecnología al servicio del docente}

En relación con la usabilidad de Telegram para el mobile learning, los alumnos perciben que es muy alta, pero que la aplicación no sustituye la labor del docente, siendo Telegram una herramienta al servicio del docente. El equipo docente es fundamental y para la mayoría de los alumnos está por encima de la aplicación. Es importante resaltar la necesidad de avanzar en el análisis de esa relación entre el docente mentor y el alumno creador de contenidos que aprende haciendo.

\subsection{El valor pedagógico de Telegram y la interrelación entre los alumnos}

Los alumnos son una parte muy importante en el análisis de la usabilidad técnica y pedagógica de Telegram, pues el $100 \%$ de ellos abordaban por primera vez un curso a través de esta herramienta y, por tanto, sus impresiones y opiniones han sido esclarecedoras.

\section{A) La interactividad}

Hay una división de opiniones entre los que valoran como suficiente el grado de interactividad que se ofrece a través del canal Telegram (recordando que solo puede escribir el docente) y los que no. Se ha demostrado que es mejor que el alumno tenga solo un interlocutor que sea quien dirija la comunicación en el aula (canal). Las opiniones de los alumnos que demandan más interacción son muy numerosas y coinciden con un perfil de estudiantes más jóvenes y de reciente matriculación; por tanto, es relevante mejorar este aspecto para este segmento de alumnos menos experimentados.

\section{B) La unidireccionalidad}

Es muy importante saber qué opinan los alumnos en relación a que sea el docente el único que pueda escribir en el canal y por tanto sea el único filtro para que los estudiantes participen o compartan sus resultados. Los alumnos más mayores y más experimentados ven la unidireccionalidad de forma muy positiva, pues ello les evita la relación con otros estudiantes menos experimentados que les frenan y hasta molestan en su formación. 


\section{C) La idea de comunidad}

Se ha demostrado que Telegram incrementa la participación de los alumnos, la interactividad con los docentes y el sentimiento de pertenencia a una comunidad que les ayuda a avanzar en su conocimiento y favorece la autoconfianza a la hora de realizar las actividades.

\subsection{El valor pedagógico de Telegram y los contenidos}

Los contenidos son el factor mejor valorado, con una nota de 8,34. Por tanto, Telegram es bueno para adquirir conocimientos teóricos y habilidades prácticas. En concreto, los más de 17.000 vídeos y fotografías son los contenidos consumidos más veces y mejor valorados.

\section{Los contenidos son el factor mejor valorado, con una nota de 8,34. Telegram es bueno para adquirir conocimientos teóricos y habilidades}

\section{A) El papel relevante de los contenidos}

Es muy importante la elaboración de materiales por parte del docente y es necesario que los propios alumnos compartan los materiales que ellos elaboran con sus actividades por el valor intrínseco de los mismos y por la motivación positiva que genera en el resto de los estudiantes. Los resultados tan positivos nos llevan a reflexionar sobre el acceso a nuevos contenidos actualizados permanentemente y a diseñar una estructura de curso que tenga como uno de sus ejes principales los contenidos audiovisuales.

\section{B) El vídeo como elemento central del contenido}

Los vídeos son el material más demandado y ofrecen una posibilidad interesante, gracias a la que se establece una mayor interacción entre el docente y el alumno sin aumentar la carga de trabajo. El uso de los contenidos audiovisuales en formato audio o vídeo ha sido determinante a la hora de valorar la usabilidad pedagógica de Telegram como herramienta de mobile learning.

\section{C) La importancia de la estructuración del contenido}

Los alumnos están muy satisfechos con el vídeo como soporte para el contenido, aunque manifiestan que, dado que se aportan más de 17.000 vídeos y contenidos audiovisuales, es necesario que exista una organización con índice temático o cronológico de los mismos. Los vídeos le dan a la formación la posibilidad de transmitir contenidos únicos, accesibles, adaptados, actualizados en tiempo real e incluso se puede acceder a ellos de forma ilimitada y reiterada. 


\subsection{El valor pedagógico de Telegram y los dispositivos}

El hecho de que Telegram funcionara como una plataforma para comunicación síncrona permitió que se pudiera diseñar un aula virtual en la que dar servicio a más de 1.000 alumnos al mismo tiempo en el curso de finanzas personales.

\author{
El uso de Telegram permitió diseñar \\ un aula virtual en la que dar servicio \\ a más de 1.000 alumnos a la vez en \\ el curso de finanzas
}

\section{A) El uso de Telegram en diferentes dispositivos de forma síncrona}

Una de las funcionalidades más importantes es que Telegram se puede utilizar de forma síncrona a través de diferentes dispositivos, como móvil, tablet, portátil o PC al mismo tiempo. El 57,60 \% de los alumnos utiliza Telegram combinado con otros dispositivos como el móvil y la pantalla del ordenador o el portátil, el $25 \%$ usa Telegram a través de móvil y el $17,40 \%$ restante lo usa en su versión ordenador o portátil.

\section{B) La posibilidad de formación blended}

El 51,80\% de los alumnos no requiere de clases presenciales. Esta circunstancia se explica porque la formación semipresencial precisa de un complemento online de igual o superior valor que la mera formación presencial y, con Telegram, se ha conseguido.

\section{C) El uso de aplicaciones complementarias a Telegram}

Es un aspecto que los alumnos valoran muy positivamente, en especial quienes están empezando o tienen preferencias por aplicaciones previamente instaladas como WhatsApp, WeChat o aplicaciones con funcionalidades de vídeo nativas, como Teams, Blackboard o Zoom.

Telegram es una herramienta óptima desde el punto de vista de la usabilidad, dado que sus funcionalidades son válidas para todo tipo de alumnos, independientemente de su edad, género o experiencia previa, tiene gran capacidad y variedad de funciones, y sirve para cualquier tipo de dispositivo.

\section{Conclusiones}

Las conclusiones del estudio son, por tanto, muy positivas, ya que Telegram se manifiesta como una magnifica herramienta para el mobile learning. Lo hace tanto en los aspectos 
relativos a la usabilidad técnica como pedagógica. En los aspectos pedagógicos, destaca por su alto grado de satisfacción en relación con el modelo de aprendizaje, el desempeño de los docentes, el empleo de contenidos audiovisuales y la utilización de multidispositivos. En términos de mejora o de adecuación del caso de estudio es interesante profundizar en la posibilidad de interacción entre los alumnos.

Para medir con más precisión el potencial de Telegram, es fundamental conocer el papel de la tecnología, de los contenidos y de los dispositivos dentro de esta relación virtual con personas. A todo ello lo llamamos, no con cierta frialdad, «usabilidad pedagógica». Por tanto, tiene sentido colaborar con este estudio e incorporar un caso más de éxito para ayudar a desarrollar metodologías para el uso de aplicaciones móviles aplicadas al mobile learning.

Mirando hacia el futuro, centraré mi nueva línea de trabajo en el estudio de la usabilidad pedagógica en relación con los tres nuevos criterios que amplían el modelo de Nokelainen. Estos tres nuevos atributos son la utilización del vídeo como tipo de contenido principal, el uso de multidispositivos indistintamente y la sincronización de dispositivos complementarios.

\section{Reflexión final}

En esta época de confinamiento, como consecuencia de la pandemia provocada por la COVID-19, se hace más presente en nuestras vidas la importancia de la comunicación entre personas y la comunicación a través de las máquinas. Hoy más que nunca dedicamos una gran parte de nuestro tiempo a comunicarnos exclusivamente por vía telemática y recíproca con nuestros seres queridos, con nuestros clientes y con nuestros alumnos.

En momentos donde el contacto físico está tasado por directrices y estrictas normas, es la tecnología la que nos facilita ese acercamiento social, pero, por ahora, solo como un maravilloso complemento que nunca podrá sustituir el contacto personal.

\section{Referencias bibliográficas}

Aldosemani, T. I. (2019). Microlearning for macro-outcomes: students' perceptions of Telegram as a microlearning tool. En T. Väljataga y M. Laanpere (Eds.), Digital Turn in SchoolsResearch, Policy, Practice (pp. 189-201). Springer.

Alkhezzi, F. y Al-Dousari, W. (2016). The Impact of Mobile Learning on ESP learners' performance. The Journal of Educators Online (JEO), 13(2), 73-101.

Brooke, J. (1996). SUS: una usabilidad «rápida y sucia». Evaluación de Usabilidad en la Industria, 189.

Davis, F. D. (1985). A Technology Acceptance Model for Empirically Testing New End-User Information Systems: Theory and Results. 
(Disertación doctoral, Massachusetts Institute of Technology).

Dewey, J. (1963). Liberalism and Social Action. Capricorn Books.

Garrote, A., Dessemontet, R. S. y Opitz, E. M. (2017). Facilitating the social participation of pupils with special educational needs in mainstream schools: a review of schoolbased interventions. Educational Research Review, 20, 12-23.

Harrison, R., Flood, D. y Duce, D. (2013). Usability of mobile applications: literature review and rationale for a new usability model. Journal of Interaction Science, 1(1), 1-43.

Heidari, J., Khodabandeh, F. y Soleimani, H. (2018). A comparative analysis of face to face instruction vs. Telegram mobile instruction in terms of narrative writing. JALT CALL Journal, 14(2), 143-156.

Iksan, Z. H. y Saufian, S. M. (2017). Mobile learning: innovation in teaching and learning using Telegram. International Journal of Pedagogy and Teacher Education, 1(1), 19-26.

Martínez Rolán, L. X., Dafonte Gómez, A. y García Mirón, S. (2017). Usos de las aplicaciones móviles de mensajería en la docencia universitaria: Telegram. VI Congreso Internacional sobre Buenas Prácticas con TIC, 18-20 de octubre de 2017. Málaga, España.

Nielsen, J. y Molich, R. (1990). Heuristic evaluation of user interfaces. Proceedings of the SIGCHI Conference on Human Factors in Computing Systems Empowering PeopleCHI'90 (pp. 249-256).

Nokelainen, P. (2004). Conceptual definition of the technical and pedagogical usability criteria for digital learning material. Proceedings of ED-MEDIA 2004.

Noor, R. M. y Hussin, N. (1970). First experience in implementing PBL for network design and management course. Journal of Problem-Based Learning, 2(1).
Pratama, H. y Prastyaningrum, I. (2019). Effectiveness of the use of integrated project based learning model, Telegram messenger, and plagiarism checker on learning outcomes. Journal of Physics: Conference Series, 1.171(1), 1-11. https://doi.org/10.108 8/1742-6596/1171/1/012033

Salas, M. A. y Subía, J. C. S. (2018). M-Learning-Una experiencia colaborativa usando el software Telegram. Revista Científica Retos de la Ciencia, 2(1), 85-94.

Sari, F. M. (2017). Maximizing Telegram application for teaching reading. 4th UAD TEFL International Conference (pp. 228-233), 9-20 de septiembre. Indonesia.

Shneiderman, B. (1996). The eyes have it: a task by data type taxonomy for information visualizations. Proceedings 1996 IEEE Symposium on Visual Languages (pp. 336-343). Washington DC, EE. UU.

Sivabalan, K. y Ali, Z. (2019). A systematic review on mobile instant messaging as a collaborative tool for language learning. International Journal of Language Education and Applied Linguistics, 9(1), 99-109.

Tullis, T. S. y Stetson, J. N. (2004). A comparison of questionnaires for assessing website usability. Usability Professional Association Conference (pp. 1-12). Mineápolis, EE. UU.

Venkatesh, V. y Davis, F. D. (2000). A theoretical extension of the technology acceptance model: four longitudinal field studies. Management Science, 46(2), 186-204.

Vygotsky, L. (1978). Interaction between learning and development. Readings on the Development of Children, 23(3), 34-41.

Xodabande, I. (2017). The effectiveness of social media network Telegram in teaching English language pronunciation to Iranian EFL learners. Cogent Education, 4(1), 1-16. https:// doi.org/10.1080/2331186X.2017.1347081 


\section{Máster en}

Dirección y Gestión de Centros Educativos

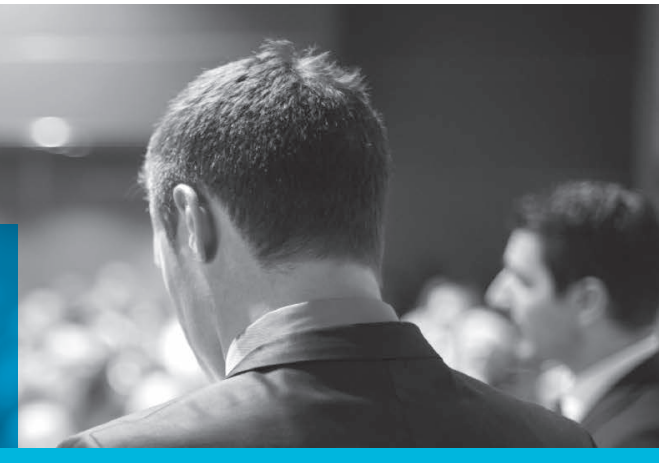

Este máster oficial [60 créditos ECTS] tiene una duración normal de 12 meses.

Los miembros de equipos directivos y los gestores de instituciones educativas encuentran en la actualidad una necesidad apremiante de formación que les habilite para dar una adecuada respuesta a las complejas situaciones que acontecen diariamente en sus lugares de trabajo. Las demandas que la sociedad actual realiza a las instituciones educativas exigen una constante renovación tanto de los centros educativos como de la formación de los profesionales que trabajan en ellos.

Dirigido a: Titulados universitarios que quieran especializarse en el ámbito de la dirección y gestión de centros educativos. Este programa formativo no exige una experiencia previa en la materia, sino que pretende proporcionar a los participantes una formación completa para la dirección y gestión de los centros de forma progresiva y eficaz.

Objetivos: Capacitar a profesionales de primer nivel para el ejercicio eficaz de la función directiva y de gestión de centros educativos. Para ello, el máster pretende dotar al alumno de las competencias y herramientas necesarias para el trabajo en un equipo multidisciplinar de profesionales, así como para promover la calidad y la innovación dentro del sistema educativo.

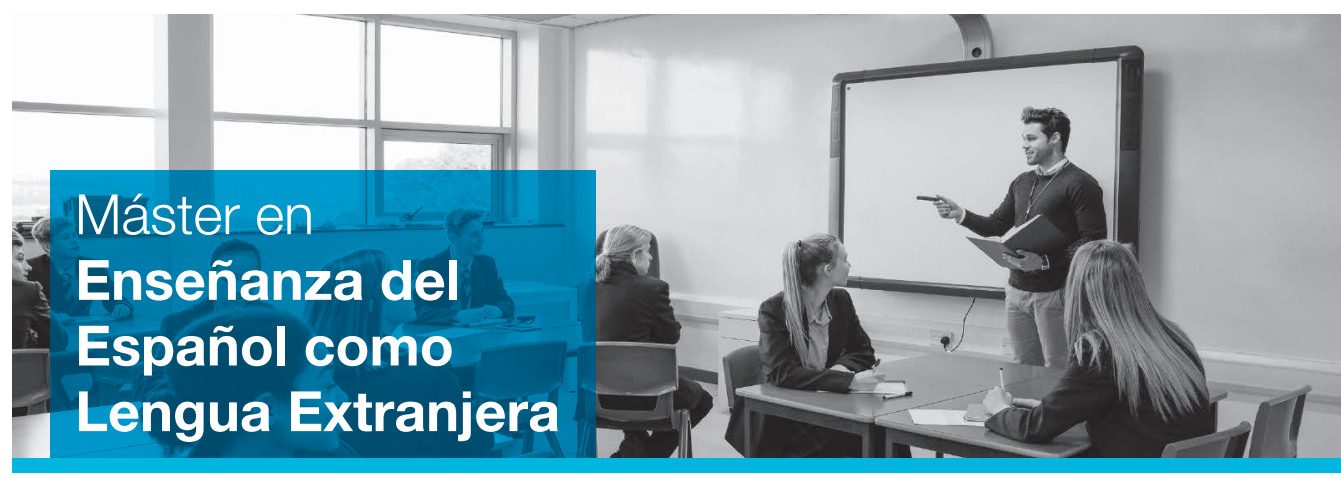

Este máster oficial [60 créditos ECTS] tiene una duración normal de 12 meses.

Dirigido a: Titulados universitarios en las distintas áreas de Lenguas Modernas, Filología, Lingüística, Traducción, Ciencias de la Educación, Humanidades, Pedagogía y Ciencias de la Comunicación y profesionales de las lenguas que deseen especializarse en la enseñanza del español como lengua extranjera.

Objetivos: Formar profesionales cualificados en el ámbito de la enseñanza del español como lengua extranjera, dotándolos de un perfil práctico y competitivo que los capacite para realizar adecuadamente y con garantía de éxito su labor profesional, facilitándoles su integración en un mercado laboral de enorme proyección internacional. 


\section{Nuestro sistema de enseñanza}

\section{/ Adaptados al mercado laboral. Adaptados a ti}

\section{Mucho más que una universidad a distancia}

La Universidad a Distancia de Madrid, UDIMA, es una institución educativa pensada y diseñada para cubrir las necesidades de las personas del siglo XXI: profesionales que demandan una universidad abierta y flexible, y que permita compatibilizar el estudio con las peculiaridades de cada estudiante, que buscan obtener una titulación universitaria reconocida oficialmente y de prestigio, adaptada a Europa y en contacto con el mundo de la empresa, y que facilite, además, una buena inserción laboral o mejore la que ya se posee.

\section{Campus virtual y sistema de evaluación}

El proceso de aprendizaje se desarrolla a través de las aulas virtuales de la universidad. Los estudiantes establecen una comunicación directa con sus profesores a través de los foros, el teléfono y otras herramientas telemáticas, como las clases en videoconferencia. Un sistema de evaluación continua, que utiliza las últimas herramientas tecnológicas en el ámbito de la didáctica, nos permite desarrollar una metodología activa que ayuda a nuestros estudiantes a «aprender haciendo».

\section{Profesorado}

En la UDIMA, la actuación de los docentes no se limita a la enseñanza, sino que también son «guias y facilitadores». La realización de un seguimiento académico pormenorizado y la personalización de la acción docente hacen de la UDIMA una comunidad de aprendizaje centrada en las personas.

\section{Materiales de enseñanza}

Nuestra editorial técnica se encarga de diseñar materiales específicamente creados para el aprendizaje online. Además, utilizamos recursos audiovisuales y material complementario de todo tipo que permiten aprovechar al máximo la experiencia formativa.

\section{Actividades de aprendizaje}

Los estudiantes van adquiriendo conocimientos a través de distintas actividades, tanto individuales como en grupo, para ir afianzando los contenidos.

\section{Test de autoevaluación}

Pruebas de evaluación online tipo test que, a modo de cuestionarios de autocomprobación, permiten que el estudiante pueda constatar los conocimientos adquiridos en el estudio previo de las unidades didácticas correspondientes.

\section{Actividades de evaluación continua}

Este tipo de actividades didácticas son pruebas de evaluación de tipo práctico: casos y trabajos basados en la búsqueda de información, el análisis de situación y la realización y presentación de informes.

\section{Exámenes presenciales}

Los exámenes finales semestrales son presenciales y con carácter obligatorio. Este tipo de prueba de evaluación permite verificar el cumplimiento de los objetivos de aprendizaje previstos en cada asignatura.

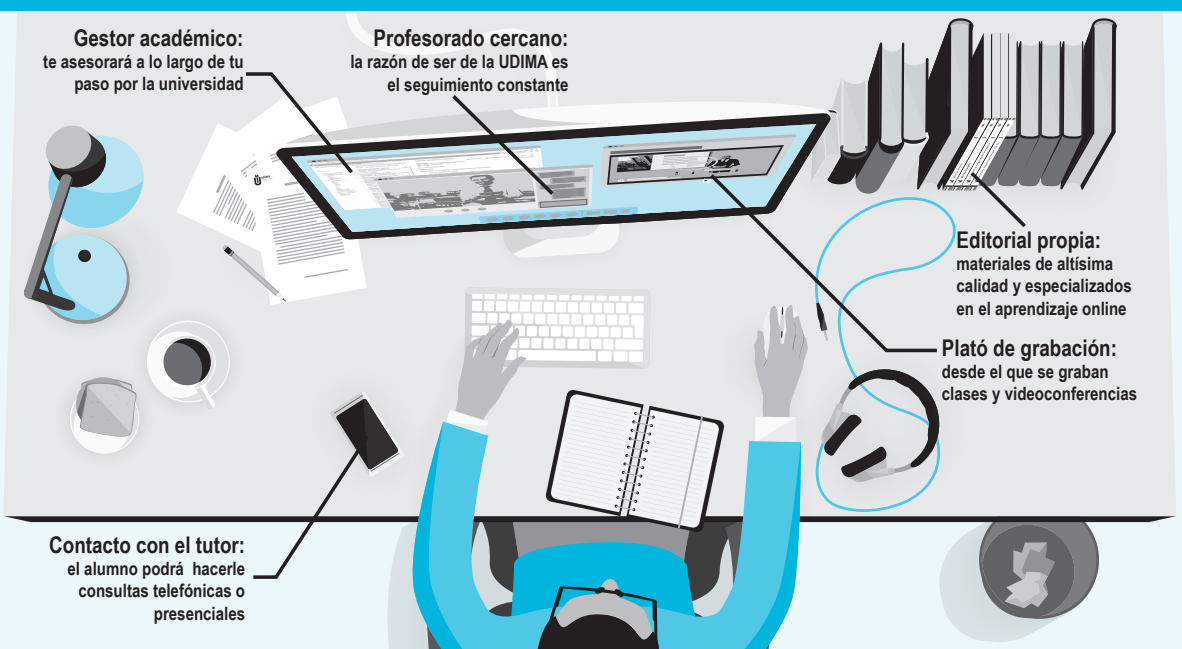

